\title{
Filament studies for laser spectroscopy on lawrencium
}

\author{
Tobias Murböck ${ }^{1,2,3}$ (D) . Sebastian Raeder ${ }^{1,2}$. Premaditya Chhetri ${ }^{1,2}$. \\ Katerine Diaz ${ }^{1,2} \cdot$ Mustapha Laatiaoui $^{1,2}$. Francesca Giacoppo ${ }^{1,2} \cdot$ Michael Block $^{1,2,4}$
}

Published online: 3 March 2020

(C) The Author(s) 2020

\begin{abstract}
The sensitive RAdiation Detected Resonance Ionization Spectroscopy (RADRIS) technique enabled the study of the atomic structure of the element nobelium (No, $Z=102$ ) for the first time. The prospect of accessing the next element, lawrencium $(\mathrm{Lr}, Z=103)$, depends on the efficiency and speed of evaporation of sample atoms from a filament catcher. To determine the desorption properties with respect to the requirements for the RADRIS technique, an off-line set-up to characterize filament catchers was developed. Using the iso-electronic homologue lutetium ( $\mathrm{Lu}, Z=71$ ) different filament materials were studied with respect to the required desorption temperatures and the background from surface ionization.
\end{abstract}

Keywords Lawrencium · Laser spectroscopy

\section{Laser spectroscopy of heavy elements with RADRIS}

Due to relativistic effects in the atomic electron shell of heavy atomic systems, e.g., the configuration of the atomic ground state in lawrencium ( $\mathrm{Lr}, Z=103)$ is expected to differ from the iso-electronic homologue lutetium $(\mathrm{Lu}, Z=71)$ [18]. A precise determination of the first ionization potential or a hyperfine structure measurement will help to unambiguously characterize the atomic ground state of $\mathrm{Lr}$, that has only been inferred indirectly. To probe the atomic shell structure of the heaviest actinides with $Z>100$, the sensitive RAdiation Detected Resonance Ionization Spectroscopy (RADRIS) technique [10] is applied at the SHIP velocity filter [15] at the GSI facility in Darmstadt, Germany. Here, the nuclei of

This article is part of the Topical Collection on Proceedings of PLATAN 2019, 1st International Conference, Merger of the Poznan Meeting on Lasers and Trapping Devices in Atomic Nuclei Research and the International Conference on Laser Probing, Mainz, Germany 19-24 May 2019 Edited by Krassimira Marinova, Michael Block, Klaus D.A. Wendt and Magdalena Kowalska

Tobias Murböck

tmurboeck@triumf.ca

1 GSI, Gesellschaft für Schwerionenforschung GmbH, Planckstr. 1, Darmstadt, Germany

2 Helmholtz-Institut Mainz, 55128, Mainz, Germany

3 Present address: TRIUMF, 4004 Wesbrook Mall, Vancouver, BC V6T 2A3, Canada

4 Institut für Kernchemie, 55128 Mainz, Germany 
interest are produced in fusion evaporation reactions, separated from the primary beam and transported to a gas cell, where they are stopped in about 95 mbar of argon gas. Within a typical experimental cycle for a nuclide with a half-life of about $1 \mathrm{~min}$, the ions are collected for $30 \mathrm{~s}$ onto a filament and evaporated by a heat-pulse of $300 \mathrm{~ms}$ duration as neutral atoms. Resonant laser ionization creates ions, which are guided to a detector where their alpha decay is registered. Using this technique laser spectroscopy of nobelium isotopes has been successfully performed [7, 17]. More technical details can be found in [2,10].

To extend this technique to the next heavier element $\mathrm{Lr}$, the release of the captured recoils from the filament is crucial. Here the fusion products must be released in a short time as neutral atoms to allow optical probing with the efficient resonance ionization method. The nobelium experiment used a tantalum filament for neutralization and evaporation, but first tests indicated that an elevated release temperature is needed for Lr. This in combination with the reduced first ionization potential (IP) compared to nobelium [18] results in a surface ion background, which may hamper the experiment sensitivity. For evaporation, the desorption enthalpy and the filament work function crucially determine the efficiency for evaporation and for the creation of surface ions, respectively. In this contribution, an off-line setup to study the desorption of lanthanide elements from different filaments is presented. In particular the desorption of lutetium from different surfaces is discussed with regard to the prospects of laser-spectroscopic investigations of the heaviest actinides.

\subsection{The evaporation process in the RADRIS setup}

In the RADRIS measurements the filament temperature is chosen in a way to guarantee complete evaporation of the atoms within the $300 \mathrm{~ms}$ heating pulse. For nobelium and radioactive ytterbium isotopes adsorbed onto a tantalum filament a temperature of about $1300 \mathrm{~K}$ was found to be sufficient for the desorption $[6,10]$. Such a low temperature helps to prolong the filament lifetime and, more importantly, to reduce the non-resonant background from ions, which are released from the filament surface during the heating pulse. The rate of these surface ions $n_{+}$relative to the rate of released neutral atoms $n_{0}$ is expressed by the Saha-Langmuir-equation

$$
\frac{n_{+}}{n_{0}}=\frac{g_{+}}{g_{0}} \exp \left(\frac{\Phi-E_{\mathrm{IP}}}{k_{B} T}\right)
$$

where the degeneracies of states for the ion and atom are give by $g_{+}$and $g_{0}$, respectively. $\Phi$ denotes the filament's work function, $E_{\mathrm{IP}}$ the atom's ionization potential, $k_{B}$ the Boltzmann constant, and $T$ the filament temperature. As a consequence, and in order to minimize the background of surface ions, the work function $\Phi$ has to be significantly lower than the atom's IP $\left(E_{\mathrm{IP}}\right)$. This is especially important for lawrencium, because it has a much lower IP compared to No [18]. In addition, the presence of an outermost $p$-electron in the atomic configuration of Lr may necessitate higher temperatures for desorption, as was shown for the homologue lanthanide series desorbing from tantalum and tungsten [12, 13].

\section{Experimental setup}

An offline setup consisting of separated preparation and detection chambers under UHV conditions ( $p=3 \times 10^{-7}$ mbar) has been used to investigate the evaporation properties of different types of filaments (Fig. 1). The preparation section contains the filament and a $\mathrm{Lu}$ source for filament coating. An UV-transparent window provides the possibility to monitor the filament temperature and to perform resonant two-step photo ionization of neutral $\mathrm{Lu}$ 


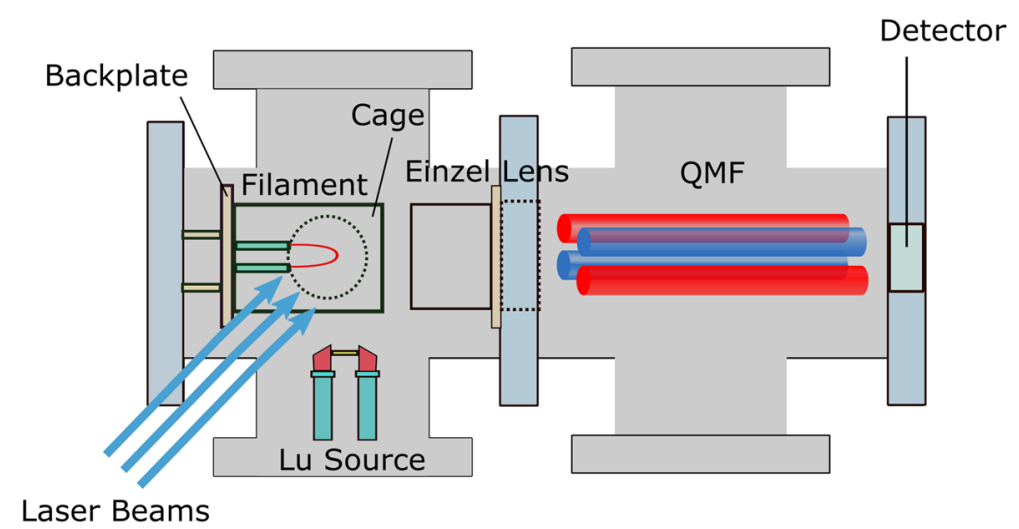

Fig. 1 Experimental setup for offline evaporation and laser ionization of lutetium in two CF160 cross pieces

atoms. The detection section comprises a channel electron multiplier (CEM) for single ion counting and a quadrupole mass filter (QMF) for background suppression and ion transport.

The $\mathrm{Lu}$ metal vapor source for coating of the filament is built from a $5 \mathrm{~mm}$ wide strip of tantalum foil of $d=0.015 \mathrm{~mm}$ thickness, which is folded around a piece of Lu foil that is $d=0.1 \mathrm{~mm}$ thick. When a heating pulse is applied across the foil, the embedded lutetium evaporates slowly and forms a neutral-metal vapor that diffuses in all directions. The filament is placed in a distance of a few centimeters from the metal vapor source in a toroidal aluminum cage. This cage is equipped with two holes: one towards the Lu source to allow coating of the filament, and a second one towards the UV-transparent window for optical access for the pyrometer and the laser beams. A backplate serves as a support for the filament and is electrically isolated from the cage, so that individual bias potentials can be applied to the cage and the backplate to create an electric gradient guiding the photo ions towards the segmented einzel lens.

For the creation of lutetium photo ions a resonant two-step scheme is applied, starting from the $5 \mathrm{~d} 6 \mathrm{~s}^{2}{ }^{2} \mathrm{D}_{3 / 2}$ ground state, and populating first the $5 \mathrm{~d} 6 \mathrm{~s}(3 \mathrm{D}) 6 \mathrm{p}^{2} \mathrm{D}_{3 / 2}^{\circ}$ state at $22124.8 \mathrm{~cm}^{-1}$ [5]. The second and actual photo-ionization is achieved in a subsequent step by exciting the electron with laser light at a photon energy of $25566.7 \mathrm{~cm}^{-1}$ into an autoionizing state. The laser system consists of two dye lasers pumped by an $\mathrm{Xe}: \mathrm{Cl}$ excimer laser operated at a repetition rate of $100 \mathrm{~Hz}$ [10]. The laser beams for both steps are transported through optical fibers to the setup, where they enter the vacuum chamber through the UV-transparent window. Spatial overlap of the laser beams with the filament is achieved with a multi-fiber coupler described in Ref. [9]. The $100 \mathrm{~Hz}$ system limits the duty cycle on the evaporated atoms and the resonantly created ions arrive at the detector in bunches of $200 \mu$ s duration. Therefore a $250 \mu$ s gate synchronized to the start of the laser pulse can be used to increase the sensitivity on laser ions, which is of particular importance for filaments with a large work function. The quadrupole mass filter (QMF) separates the detector from the preparation section and was operated with a mass resolution of about $10 \mathrm{u}$. This resolving power is sufficient to suppress any background from molecular or alkaline ions and allows at the same time a high transmission towards the detector.

A typical measurement cycle consists of two steps (Fig. 2): first, the filament is coated with $\mathrm{Lu}$ from the source, and second, the coating process is stopped and the filament is resistively heated to a fixed temperature to evaporate $\mathrm{Lu}$ from its surface. The heating pulse 


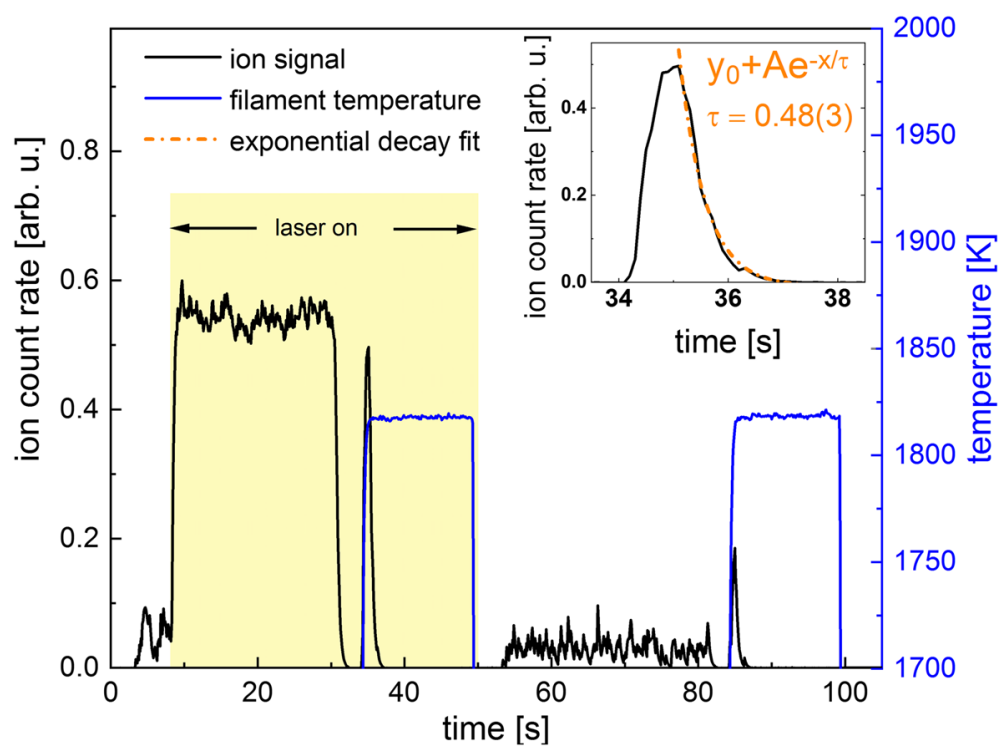

Fig. 2 Typical ion count rate (left scale) and filament temperature (right scale) as recorded during a measurement cycle. Strong signals can e.g. be seen during the heating of the Lu source $(2 \mathrm{~s} \leq t \leq 32 \mathrm{~s})$ and the heating of the filament ( $34 \mathrm{~s} \leq t \leq 50 \mathrm{~s})$. See text for more details

applied during the second step is chosen long enough to guarantee that the filament is free from any adsorbed material before the measurement cycle is repeated. A comparison of the detected ion rates with and without resonant laser ionization, respectively, allows the fraction of ions produced by surface ionization to be determined. In addition, the time needed for desorption is monitored while monitoring the ion signal in the course of the time. The filament temperature is monitored with a pyrometer (Luma-Sense Tech., Impac IS 6 Advance).

\section{Filament characterization}

An ion catcher filament in the RADRIS experiment must sustain the temperature needed for desorption without creating background by non-resonant surface ions. In addition, a low desorption temperature is favorable, as strong filament heating introduces turbulence in the gas cell, which distributes the released atoms in a larger volume and negatively impacts the efficiency of the RADRIS method [9].

The requirement of a high temperature durability suggests the use of refractory metals (e. g. Hf, Ta, $\mathrm{W}$ or $\mathrm{W}_{99.4} \mathrm{Th}_{0.6}$ ), which are commercially available, or filaments of refractory metals with an additional sputter-coated layer (typically $1-10 \mu \mathrm{m}$ ) to tailor the filament's surface properties. Table 1 summarizes the work functions reported for different materials as well as the IP values for nobelium, lawrencium, ytterbium and lutetium. According to (1), for a low surface ionization yield the respective filament or surface material must have a low work function. At a temperature of $1500 \mathrm{~K}$, for instance, we expect the yield of surface ions to increase for $\mathrm{Lr}$ with an IP of $4.96 \mathrm{eV}$ by more than four orders of magnitude compared to No with an IP of $6.63 \mathrm{eV}$, if the same filament material was used. 
Table 1 Comparison of the work functions $\Phi$ and the melting temperatures $T_{\text {melt }}$ for a selection of filament materials and the ionization potentials (IP) for some elements of interest

\begin{tabular}{lllll}
\hline Filament & $\Phi(\mathrm{eV})^{\mathrm{a}}$ & $T_{\text {melt }}(\mathrm{K})$ & Element & IP $(\mathrm{eV})$ \\
\hline Hf & 3.9 & 2506 & $\mathrm{Yb}$ & $6.25[1]$ \\
Ta & 4.25 & 3290 & $\mathrm{Lu}$ & $5.42[11]$ \\
W & 4.55 & 3695 & $\mathrm{No}$ & $6.63[3]$ \\
Th & 3.4 & 2028 & $\mathrm{Lr}$ & $4.96[19]$ \\
Y & 3.1 & 1799 & & \\
\hline
\end{tabular}

Commercially available wires with high elemental purity have been used in case of the refractory metals $\mathrm{Hf}$, $\mathrm{Ta}$, and $\mathrm{W}$. Thoriated tungsten wires and yttrium coated tungsten wires have been used for the metals with lower melting point. Since the work function of a surface can be influenced by oxygen poisoning, the values given here will set a lower limit for the values under realistic vacuum conditions

a from [14] for polycrystalline specimens
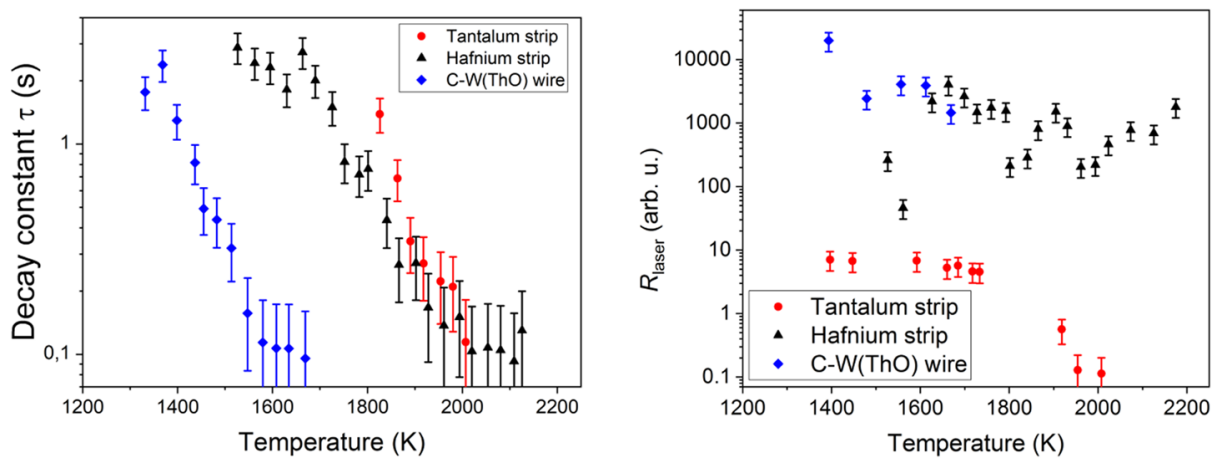

Fig. 3 Left: Decay constant (desorption time) as a function of the filament temperature. Right: Ratio of laser photo ions to surface ions as function of the filament temperature

\subsection{Desorption time of lutetium and fraction of surface ions}

To quantify the desorption process, an exponential decay has been fitted to the data obtained during filament heating (see inset of Fig. 2), from which the characteristic desorption time $\tau$ and the temperature $T$ were extracted. Figure 3 (left) shows the dependence of the decay constant $\tau$ on the measured filament temperature. As expected, the desorption becomes faster with higher temperatures. From the presented results the $\mathrm{C}-\mathrm{W}(\mathrm{ThO})$ filament in the geometry of a $0.1 \mathrm{~mm}$ wire with a $1 \mu \mathrm{m}$ carbon layer showed the lowest desorption temperatures, while for Hf and Ta strips $(1 \mathrm{~mm} \times 25 \mu \mathrm{m})$ higher temperatures are required. This strip geometry was chosen for Hf, as Hf wires of $0.1 \mathrm{~mm}$ thickness got ductile and deformed strongly at temperatures exceeding $1200 \mathrm{~K}$. It should be mentioned, however, that the use of different filament geometries introduced a systematic deviation of the measured desorption temperatures. This effect is attributed to the difference in the geometrical cross sections as seen by the pyrometer. In the case of tantalum the desorption temperature for a strip and a $0.12 \mathrm{~mm}$ filament wire differed by about $200 \mathrm{~K}$. As these measurements present a preparation for laser spectroscopy, where similar temperature measurement procedures 

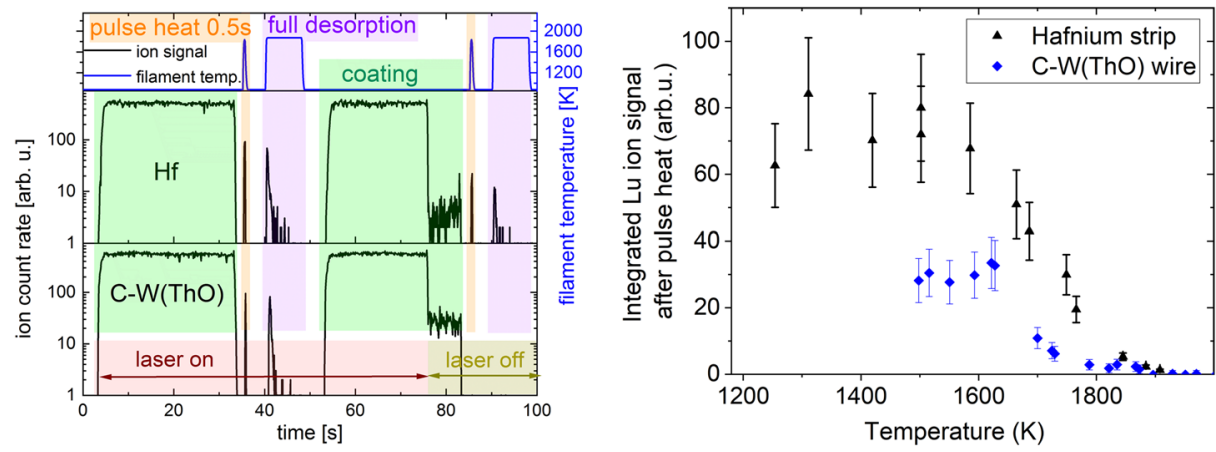

Fig. 4 Left: The measurement scheme for Lu desorption by filament pulse heating. After a period of filament coating $(4 \mathrm{~s} \leq t \leq 34 \mathrm{~s})$ a $0.5 \mathrm{~s}$ short heat pulse $(t \approx 36 \mathrm{~s})$ partially desorbs the adsorbed Lu. A longer heating period ( $40 \mathrm{~s} \leq t \leq 49 \mathrm{~s}$ ) follows to fully desorb the remaining Lu from the filament. For $t \geq 50 \mathrm{~s}$, these steps are repeated to determine the surface ion component of the signal. Right: The residual signal detected during the second the heating period as a function of the filament peak temperature for a $\mathrm{Hf}$ and a $\mathrm{C}-\mathrm{W}(\mathrm{THO})$ filament

will be applied, this systematic shift does not impair our conclusions, but would have to be considered for the determination of the desorption enthalpies.

In addition, the ratio of laser-ions to surface-ions

$$
R_{\text {laser }}=\frac{N_{\text {photo ions }}}{N_{\text {surface ions }}}=\frac{N_{\text {laser On }}-N_{\text {laser Off }}}{N_{\text {laser Off }}}
$$

was evaluated for the different filaments. The results are shown in Fig. 3 (right). The ratio scatters on a large scale, because the ion signal depends on many parameters such as the electrostatic potentials, the laser beam alignment, and the laser power. The uncertainty was determined from the scatter of several measurements and reflects the influence of these different effects. The results still allow a comparison of the different filaments: tantalum clearly produces a majority of surface ions while the $\mathrm{C}-\mathrm{W}(\mathrm{ThO})$ filament possesses the lowest ion background, followed by the Hf filament.

\subsection{Pulse heating of the filament}

The data presented so far were obtained by heating the filament for several seconds with a constant heating current, thus allowing the filament temperature to equilibrate and to determine the desorption time and the laser-ion to surface-ion ratio under controlled conditions. However, during the RADRIS experiment the filament will be pulse heated and the peak temperature resulting in a complete desorption has to be determined in a dedicated measurement. To this end, a $0.5 \mathrm{~s}$ short heat pulse was applied to the filament followed by a longer heating period to fully desorb the adsorbed lutetium from the filament as shown in the left panel of Fig. 4. Due to limitations in the data acquisition for very fast signals, the depletion of the slower signal served as a measure for the desorption during the short pulse, rather than determining the signal produced during the short heat pulse directly. The results are shown in the right panel of Fig. 4. For Hf filaments complete Lu desorption was found for peak temperatures of $1800 \mathrm{~K}$, while for $\mathrm{C}-\mathrm{W}(\mathrm{ThO})$ filaments a slightly lower temperature of $1700 \mathrm{~K}$ was sufficient. The lower desorption temperature for $\mathrm{C}-\mathrm{W}(\mathrm{ThO})$ is in agreement with the finding that this filament type had the shortest desorption time of all materials under investigation (Fig. 3 (left)). 


\subsection{Filament longevity and performance stability}

During RADRIS measurements, a filament will undergo about 3000 heating pulses per day. While the tantalum filaments have proven very reliable for long-term operation up to one week (20 000 cycles), extended testing showed that hafnium filaments last for more than 3600 cycles before breaking. The longevity of the Hf filaments was substantially reduced when they were exposed to air for a longer time, supposedly due to the formation of a hafnium oxide layer that can extend over a significant depth of the $25 \mu \mathrm{m}$ thin $\mathrm{Hf}$ foil. The C-W(ThO) filaments lasted for typically 1000 cycles. In contrast, the C-W(ThO) filaments did not break but lost their desired properties due to the depletion of the sputter-coated carbon layer. Furthermore, variance in the sputter coating process resulted in quite some scatter in the performance of the individual filaments. While these filaments possess in principle desirable properties, a more developed preparation process would be needed to achieve the required reliability for an on-line experiment.

\subsection{Additional filaments}

For pure tungsten, for which data on the desorption of Lu from $\mathrm{W}$ surfaces is reported [13], almost exclusively surface ions and barely any photo ions were observed. Thoriated tungsten filaments are reported to have a low work function after a process of activation, which results in the formation of a thorium layer at the surface of the filament. During the activation, the filaments are first heated to a temperature of more than $2200 \mathrm{~K}$, at which the $\mathrm{ThO}_{2}$ in the bulk of the filament is reduced to metallic thorium. At temperatures of 1900$2100 \mathrm{~K}$ the filament can be operated for thousands of hours in an equilibrium between the evaporation of metallic thorium from the surface and the replenishing of the surface layer by thorium diffusion from the bulk [8]. However, first tests with a $\mathrm{W}(\mathrm{ThO})$ filament $(0.1 \mathrm{~mm}$ wire, $0.6 \% \mathrm{ThO}$ content) resulted in a large fraction of surface ions, not different from the one obtained from pure W. In literature is was found that a partial oxygen pressure of only $10^{-15}$ mbar can already poison the surface properties [4]. To operate in higher partial pressures of up to $10^{-6}$ mbar, it is recommended that a tungsten carbide $\mathrm{W}_{2} \mathrm{C}$ layer is formed with usually a tenth of the wire radius in thickness $[4,20]$. The presence of carbon in the surface counteracts the oxygen adsorption by the formation of volatile $\mathrm{CO}$. Therefore, the $\mathrm{C}-\mathrm{W}(\mathrm{THO})$ filaments as described in the previous sections were sputter coated with a carbon layer of about $1 \mu \mathrm{m}$ thickness. An alternative approach to grow a tungsten carbide layer by heating the filament in an atmosphere of hydrogen and hydrocarbon gas [16, 20] has been tried but proved ultimately difficult to implement for on-line experiments. Furthermore, we tested tantalum and tungsten filaments with coatings of low work function materials such as $\mathrm{LaB}_{6}, \mathrm{ZrC}$, and $\mathrm{Y}$ (all layers produced by sputter coating), but in all the cases the coatings evaporated at temperatures lower then the release temperature of $\mathrm{Lu}$.

\section{Summary and outlook}

With the goal of performing laser spectroscopy on lawrencium utilizing the RADRIS technique, a variety of filament materials have been investigated with respect to their properties in the desorption of lutetium, the chemical homologue of lawrencium. Two promising candidates have been identified, high-purity hafnium and thoriated tungsten with an additional layer of sputter coated carbon (C-W(ThO)). Both types of filaments combine the desired properties of a low work function and a low desorption temperature, resulting in a minimum 
surface ion background and little turbulence in the buffer gas in the vicinity of the filament, respectively. While the $\mathrm{C}-\mathrm{W}(\mathrm{ThO})$ filaments had the lowest surface-ion background of all filaments under investigation, their properties also showed a larger variance compared to filaments from commercially available material. Filaments built from Hf foils gave more reliable results and still offer a reduction of the surface ion background of more than an order of magnitude compared to Ta. We are confident that under these conditions the atomic structure of lawrencium could be studied for the first time in upcoming RADRIS experiments.

Acknowledgements Open Access funding provided by Projekt DEAL. This work was supported by the Helmholtz Association. We are especially indebted to B. Lommel, B. Kindler and V. Yakusheva from the GSI target laboratory for their efforts in coating the filaments for this study.

Open Access This article is licensed under a Creative Commons Attribution 4.0 International License, which permits use, sharing, adaptation, distribution and reproduction in any medium or format, as long as you give appropriate credit to the original author(s) and the source, provide a link to the Creative Commons licence, and indicate if changes were made. The images or other third party material in this article are included in the article's Creative Commons licence, unless indicated otherwise in a credit line to the material. If material is not included in the article's Creative Commons licence and your intended use is not permitted by statutory regulation or exceeds the permitted use, you will need to obtain permission directly from the copyright holder. To view a copy of this licence, visit http://creativecommonshorg/licenses/by/4.0/.

\section{References}

1. Aymar, M., Debarre, A., Robaux, O.: Highly excited levels of neutral ytterbium. II. multichannel quantum defect analysis of odd- and even-parity spectra. J. Phys. B 13(6), 1089-1109 (1980). https://doi.org/10.1088/0022-3700/13/6/016

2. Backe, H., Lauth, W., Block, M., Laatiaoui, M.: Prospects for laser spectroscopy, ion chemistry and mobility measurements of superheavy elements in buffer-gas traps. Nucl. Phys. A 944, $492-517$ (2015). https://doi.org/10.1016/j.nuclphysa.2015.07.002

3. Chhetri, P., Ackermann, D., Backe, H., Block, M., Cheal, B., Droese, C., Düllmann, C.E., Even, J., Ferrer, R., Giacoppo, F., Götz, S., Heßberger, F.P., Huyse, M., Kaleja, O., Khuyagbaatar, J., Kunz, P., Laatiaoui, M., Lautenschläger, F., Lauth, W., Lecesne, N., Lens, L., Minaya Ramirez, E., Mistry, A.K., Raeder, S., Van Duppen, P., Walther, T., Yakushev, A., Zhang, Z.: Precision measurement of the first ionization potential of nobelium. Phys. Rev. Lett. 120(263), 003 (2018). https://doi.org/10.1103/PhysRevLett.120.263003

4. Jenkins, R.: A review of thermionic cathodes. Vacuum 19(8), 353-359 (1969). https://doi.org/10.1016/ s0042-207x(69)80077-1

5. Kramida, A., Ralchenko, Y., Reader, J.: NIST ASD Team: Nist atomic spectra database (version 5..7.1). Online (2019). https://doi.org/10.18434/T4W30F. Available at http://physics.nist.gov/asd, Accessed 11.11.2016

6. Laatiaoui, M., Backe, H., Block, M., Heßberger, F.P., Kunz, P., Lautenschläger, F., Lauth, W., Sewtz, M., Walther, T.: On laser spectroscopy of the element nobelium $(z=102)$. Europ. Phys. J. D 68(3), 1-6 (2014). https://doi.org/10.1140/epjd/e2014-40617-6

7. Laatiaoui, M., Lauth, W., Backe, H., Block, M., Ackermann, D., Cheal, B., Chhetri, P., Düllmann, C.E., van Duppen, P., Even, J., Ferrer, R., Giacoppo, F., Götz, S., Heßberger, F.P., Huyse, M., Kaleja, O., Khuyagbaatar, J., Kunz, P., Lautenschläger, F., Mistry, A.K., Raeder, S., Minaya Ramirez, E., Walther, T., Wraith, C., Yakushev, A.: Atom-at-a-time laser resonance ionization spectroscopy of nobelium. Nature 538(7626), 495-498 (2016). https://doi.org/10.1038/nature19345

8. Langmuir, I.: The electron emission from thoriated tungsten filaments. Phys. Rev. 22, 357-398 (1923). https://doi.org/10.1103/PhysRev.22.357. https://link.aps.org/doi/10.1103/PhysRev.22.357

9. Lautenschläger, F.: Laserspektroskopie an Nobelium $(Z=102)$ in Einer Puffergaszelle. Ph.D. thesis, Technische Universität Darmstadt (2016)

10. Lautenschläger, F., Chhetri, P., Ackermann, D., Backe, H., Block, M., Cheal, B., Clark, A., Droese, C., Ferrer, R., Giacoppo, F., Götz, S., Heßberger, F., Kaleja, O., Khuyagbaatar, J., Kunz, P., Mistry, A., 
Laatiaoui, M., Lauth, W., Raeder, S., Walther, T., Wraith, C.: Developments for resonance ionization laser spectroscopy of the heaviest elements at ship. Nucl. Instrum. Meth. Phys. Res. B 383, 115-122 (2016). https://doi.org/10.1016/j.nimb.2016.06.001

11. Maeda, H., Mizugai, Y., Matsumoto, Y., Suzuki, A., Takami, A.: Highly excited even Rydberg series of Lu I studied by two-step laser photoionisation spectroscopy. J. Phys. B 22(18), L511-L516 (1989). https://doi.org/10.1088/0953-4075/22/18/002

12. Medvedev, B.: Thermal desorption of lanthanide atoms from the surface of tantalum. Sov. Phys. - Solid State. 16(7), 1242-1244 (1975)

13. Medvedev, B., Ionov, N., Belyakov, Y.: Thermal desorption of lanthanide atoms and ions from the surface of tungsten. Sov. Phys.-Solid State (Engl. Transl.) 15(9), 1743-1746 (1974)

14. Michaelson, H.B.: The work function of the elements and its periodicity. J. Appl. Phys. 48(11), 47294733 (1977). https://doi.org/10.1063/1.323539

15. Münzenberg, G., Faust, W., Hofmann, S., Armbruster, P., Güttner, K., Ewald, H.: The velocity filter ship, a separator of unslowed heavy ion fusion products. Nucl. Instrum. Meth. 161(1), 65-82 (1979). https://doi.org/10.1016/0029-554X(79)90362-8

16. Okoli, S., Haubner, R., Lux, B.: Carburization of tungsten and tantalum filaments during low-pressure diamond deposition. Surf. Coat. Technol. 47(1-3), 585-599 (1991). https://doi.org/10.1016/02578972(91)90329-u

17. Raeder, S., Ackermann, D., Backe, H., Beerwerth, R., Berengut, J.C., Block, M., Borschevsky, A., Cheal, B., Chhetri, P., Düllmann, C.E., Dzuba, V.A., Eliav, E., Even, J., Ferrer, R., Flambaum, V.V., Fritzsche, S., Giacoppo, F., Götz, S., Heßberger, F.P., Huyse, M., Kaldor, U., Kaleja, O., Khuyagbaatar, J., Kunz, P., Laatiaoui, M., Lautenschläger, F., Lauth, W., Mistry, A.K., Minaya Ramirez, E., Nazarewicz, W., Porsev, S.G., Safronova, M.S., Safronova, U.I., Schuetrumpf, B., Van Duppen, P., Walther, T., Wraith, C., Yakushev, A.: Probing sizes and shapes of nobelium isotopes by laser spectroscopy. Phys. Rev. Lett. 120(232), 503 (2018). https://doi.org/10.1103/PhysRevLett.120.232503

18. Sato, T.K., Asai, M., Borschevsky, A., Beerwerth, R., Kaneya, Y., Makii, H., Mitsukai, A., Nagame, Y., Osa, A., Toyoshima, A., Tsukada, K., Sakama, M., Takeda, S., Ooe, K., Sato, D., Shigekawa, Y., Ichikawa, S., Düllmann, C.E., Grund, J., Renisch, D., Kratz, J.V., Schädel, M., Eliav, E., Kaldor, U., Fritzsche, S., Stora, T.: First Ionization Potentials of Fm, Md, No, and Lr: Verification of Filling-Up of $5 f$ Electrons and Confirmation of the Actinide Series. J. Am. Chem. Soc. 140(44), 14,609-14,613 (2018). https://doi.org/10.1021/jacs.8b09068

19. Sato, T.K., Asai, M., Borschevsky, A., Stora, T., Sato, N., Kaneya, Y., Tsukada, K., Düllmann, C.E., Eberhardt, K., Eliav, E., Ichikawa, S., Kaldor, U., Kratz, J.V., Miyashita, S., Nagame, Y., Ooe, K., Osa, A., Renisch, D., Runke, J., Schädel, M., Thörle-Pospiech, P., Toyoshima, A., Trautmann, N.: Measurement of the first ionization potential of lawrencium, element 103. Nature 520(7546), 209-211 (2015). https://doi.org/10.1038/nature14342

20. Schneider, P.: Thermionic emission of thoriated tungsten. J. Chem. Phys. 28(4), 675-682 (1958). https://doi.org/10.1063/1.1744212

Publisher's note Springer Nature remains neutral with regard to jurisdictional claims in published maps and institutional affiliations. 